\title{
TRAITEMENT D'IMAGES SATELLITAIRES À TRÈS HAUTE RÉSOLUTION SPATIALE ET IDENTIFICATION DE ZONES À ENJEUX DANS L'AMÉNAGEMENT DES TRAMES VERTES URBAINES
}

\author{
Pauline Crombette ${ }^{1}$, Sébastien Le Corre ${ }^{1}$, Claire Tinel ${ }^{2}$ \\ 1: LISST-CIEU, Toulouse, pauline.crombette@univ-tlse2.fr \\ 2: Centre National d'Études Spatiales (CNES), Toulouse
}

\begin{abstract}
Résumé
Ce travail présente la mise en place d'une chaîne de traitement d'images à très haute résolution spatiale pour l'extraction de la végétation urbaine et une mise en application méthodologique au service des aménageurs du territoire pour l'identification des connectivités écologiques, un concept politique issu des lois Grenelle de l'Environnement.

Les résultats ont montré qu'il est possible d'extraire la végétation urbaine d'une image satellite Pléiades à l'aide d'une méthode simple, semi-automatisée et utilisant des outils libres tant pour des traitements raster que vecteurs. La chaîne de traitement développée permet ainsi de distinguer la végétation basse de la végétation arborée à partir d'indices radiométriques simples et d'un filtre d'extraction des contours.

La discrimination de cette végétation urbaine nous a ensuite permis de valoriser ces résultats et de développer une méthode d'identification des connectivités écologiques urbaines qui s'appuie sur la théorie des graphes. Cette nouvelle information géographique, couplée à d'autres indicateurs et documents d'urbanisme, permet de localiser les zones d'enjeux où un besoin de maintien de la biodiversité et une volonté d'urbanisation s'affrontent.
\end{abstract}

Mots-clés : végétation urbaine, connectivités écologiques, théorie des graphes, très haute résolution spatiale

\begin{abstract}
This paper presents the implementation of a processing chain for urban vegetation extraction from very high spatial resolution images Pléiades, and a methodological application in land management to identify ecological connectivity, a political concept set up with the Grenelle laws.

Our research shows that we can use a simple and semi-automated method to obtain a distribution of grass and trees on urban environment using Pléiades images, free tools image processing and GIS software. The process is based on radiometric indices and edge extraction filter.

A second phase of this project focuses on the identification of urban ecological connectivity using graph theory. The urban vegetation associated with others indicators and planning documents is used to locate conflict areas: between biodiversity preservation and urbanization.
\end{abstract}

Keywords: urban vegetation, ecological continuities, graph theory, very high spatial resolution

\section{Introduction}

Les résultat des discussions tenues à l'occasion des lois Grenelle de l'Environnement I et II en 2007 et 2010, ont abouti au concept de Trame verte et bleue (TVB) qui désigne à la fois une réalité écologique et un ensemble de mesures destinées à mieux prendre en compte cette réalité dans les politiques d'aménagement du territoire. Face à un constat de fragmentation grandissant des espaces naturels, conséquence de l'étalement urbain diffus, chaque intercommunalité se doit désormais d'intégrer dans ses documents de planification une réflexion écologique favorisant la biodiversité et permettant les flux d'espèces animales et végétales via un réseau écologique.

Des travaux de recherche sur l'identification de ces continuités écologiques sont actuellement menés dans les milieux ruraux et naturels, mais une simple transposition de ces modèles et méthodes aux espaces urbains n'est pas satisfaisante en raison de certaines spécificités (Clergeau et Hubert-Moy, 2011). Parallèlement, les connaissances écologiques actuelles ne permettent pas encore d'évaluer de façon certaine la fonctionnalité effective des corridors urbains. Dans un contexte de relative incertitude, rétablir ou pérenniser une connectivité entre ces habitats apparaît cependant important pour le maintien de la biodiversité sur les territoires artificialisés soumis à de rapides changements.

Par ailleurs, ces mêmes territoires sont régis par une autre réglementation. Celle-ci vise à une gestion économe de l'espace en luttant contre l'étalement urbain et s'inscrit dans la loi relative à la Solidarité et au Renouvellement Urbains (SRU) du 13 décembre 2000, confortée par la loi Grenelle II. II s'agit, par des instruments de planification urbaine (Schéma de Cohérence Territoriale (SCoT)) et des réglementations locales d'urbanisme (Plan local d'urbanisme (PLU)), d'inciter aux remplissages des dents creuses, les espaces non bâtis entourés de parcelles bâties.

C'est dans ce double contexte que les services de l'État comme les collectivités territoriales ont besoin de données et de méthodes pour identifier localement les zones combinant une forte pression urbanistique et une biodiversité à préserver, qu'elle soit sous la forme de réservoirs d'habitats végétalisés ou bien de connectivités écologiques (corridors).

Nous ne nous intéressons qu'à la composante verte du concept de TVB, c'est-à-dire la végétation. Spatialement, la gestion de ces éléments nécessite 
donc de pouvoir les décrire et analyser leur distribution, en s'attachant à leur aspect structurel (forme et agencement spatial des milieux) et dans la mesure du possible à leur aspect fonctionnel (caractéristiques écologiques ou biologiques de ces milieux) lorsque les données sont disponibles (Baudry et Merriam, 1988; Uezu et al., 2005). La représentation cartographique est alors indispensable pour la planification de mesures quelle que soit l'échelle considérée, d'un réseau international et européen à un niveau départemental puis local à l'aide des SCoT ou des PLU.

Le but de cette recherche est d'évaluer la faisabilité d'une première cartographie de la végétation urbaine à partir d'imagerie satellitaire à très haute résolution spatiale de type Pléiades. Cette donnée cartographique est ensuite exploitée dans un contexte d'urbanisation rapide pour identifier les continuités écologiques et localiser les espaces à enjeux, entre maintien de la biodiversité et volonté de densification des espaces.

\section{Le territoire d'étude}

Cette étude a d'abord été menée sur la commune de Toulouse en Haute-Garonne (31). On y observe de nombreux espaces végétalisés où des changements d'occupation des sols (OCS) s'opèrent inévitablement d'une année sur l'autre. La méthodologie déployée se doit d'être robuste, simple et mobilisant des outils libres tout en étant transposable à d'autres espaces. Raisons pour laquelle d'autres sites sont en cours d'études comme les communes de Muret (31), de PierrefitteNestalas(65) ainsi que la ville de Strasbourg (67). Toutefois, cet article ne présente que les résultats obtenus sur la commune de Toulouse.

\section{Données et outils}

Pour répondre à nos questions de recherche, nous avons eu recours à deux types de données.

D'une part, nous avons utilisé des images satellite à haute et très haute résolution spatiale, provenant des capteurs Pléiades et SPOT-5. Le satellite Pléiades fournit des images panchromatiques et mutlispectrales de résolution spatiale respectives de $70 \mathrm{~cm}$ et $2 \mathrm{~m} 80$. Pour la mise en place de la chaîne de traitement d'extraction de la végétation urbaine, nous avons utilisé une image Pléiades- $1 \mathrm{~A}$ acquise sur la commune de Toulouse, le 07 mai 2012 selon un angle de vue de $22^{\circ}$. Elle couvre une superficie d'environ $490 \mathrm{~km}^{2}$. L'Institut national de l'information géographique et forestière (IGN) a orthorectifiée cette image. La première étape d'extraction de la végétation a été réalisée sur la totalité de l'image Pléiades. En revanche, la seconde phase d'identification des connectivités écologiques n'a été menée que sur une zone test de $9 \mathrm{~km}^{2}$ au nord de Toulouse, illustrée par l'encadré rouge sur la figure 1. L'image SPOT-5 orthorectifiée est utilisée dans une optique de comparaison et d'évaluation des résultats de notre classification. II s'agit d'une image multispectrale de $10 \mathrm{~m}$ de résolution spatiale. Acquise le 03 mai 2012 selon un angle de vue de $23^{\circ}$, elle couvre un territoire d'environ $4200 \mathrm{~km}^{2}$, mais ne recouvre pas entièrement l'emprise de l'image Pléiades (figure 1).

D'autre part, nous avons utilisé des données vectorielles dans les phases d'apprentissages pour l'étape de classification en nous appuyant sur la couche "Végétation » de la BD TOPO ${ }^{\circledR}$ de I'IGN. La donnée disponible sur le département de la Haute-Garonne ne permet pas de distinguer la nature de la végétation. L'ensemble des objets y sont classés en «Zone arborée " pour une dernière mise à jour au 24/07/2006.

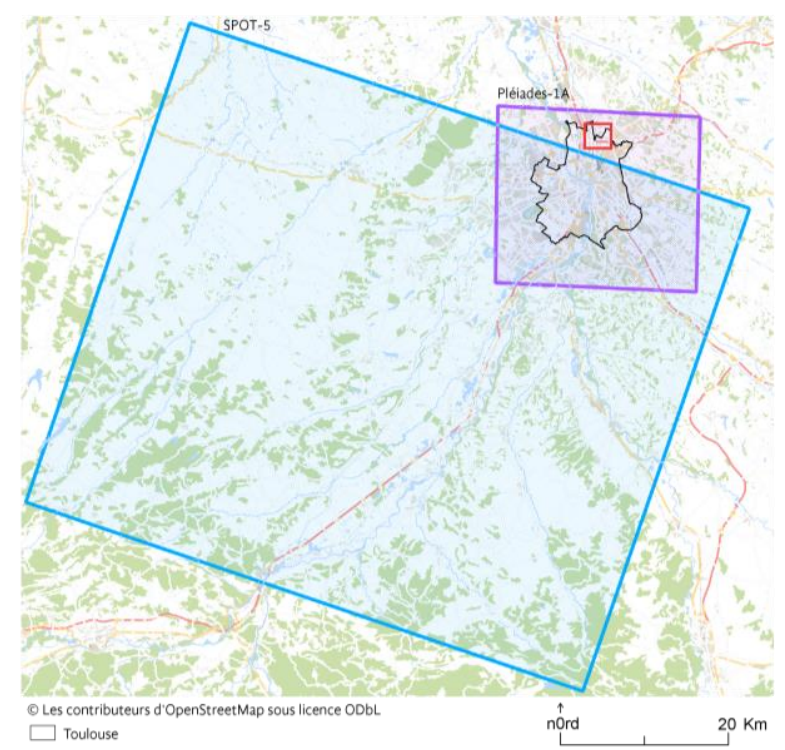

Figure 1 : Emprise des images Spot-5 (encadré bleu) et Pléiades-1A (encadré violet), et de la zone de test pour l'identification des connectivités écologiques (encadré rouge)

Afin de répondre aux préconisations de l'État et dans une volonté de large diffusion méthodologique au sein des collectivités et auprès des acteurs du territoire, les outils utilisés pour cette recherche sont des outils libres. Le traitement d'images est réalisé à l'aide de la bibliothèque OrfeoToolBox (OTB) (http://orfeotoolbox.org). Le choix de l'OTB se justifie par un gage de pérennité, le CNES étant l'acteur majeur du développement de cet outil.

L'utilisation du Système d'Information Géographique (SIG) QGIS s'est lui aussi imposé dans la continuité d'un développement libre (http://qgis.org). Cette première chaîne de traitement se veut aussi la plus automatisée possible, afin de faciliter son appropriation et son exploitation. Pour cette raison, et dans la mesure du possible, les traitements sont donc lancés via des scripts ou des modèles sous QGIS qui ont été développés spécifiquement pour cette étude. Le volume de données étant potentiellement conséquent en raison notamment de la très haute résolution spatiale, les traitements sont mobilisables à façon sur l'ensemble de la scène ou sur un ensemble de dalles.

L'identification et l'évaluation des connectivités écologiques mobilisent un troisième logiciel de modélisation des réseaux écologiques par les graphes paysagers, Graphab (http://thema.univfcomte.fr/productions/graphab/).

\section{Chaîne de traitement d'extraction de la végétation urbaine}

Notre chaîne de traitement pour l'identification de la végétation urbaine est présentée sur la figure 2. Les entrées de cette chaîne sont des images satellites optiques Pléiades à très haute résolution spatiale en mode multispectrale $(2,7 \mathrm{~m})$ et en mode panchromatique $\quad(0,7 \mathrm{~m})$ rééchantillonnée à $2 \mathrm{~m}$ et $0,5 \mathrm{~m}$. 


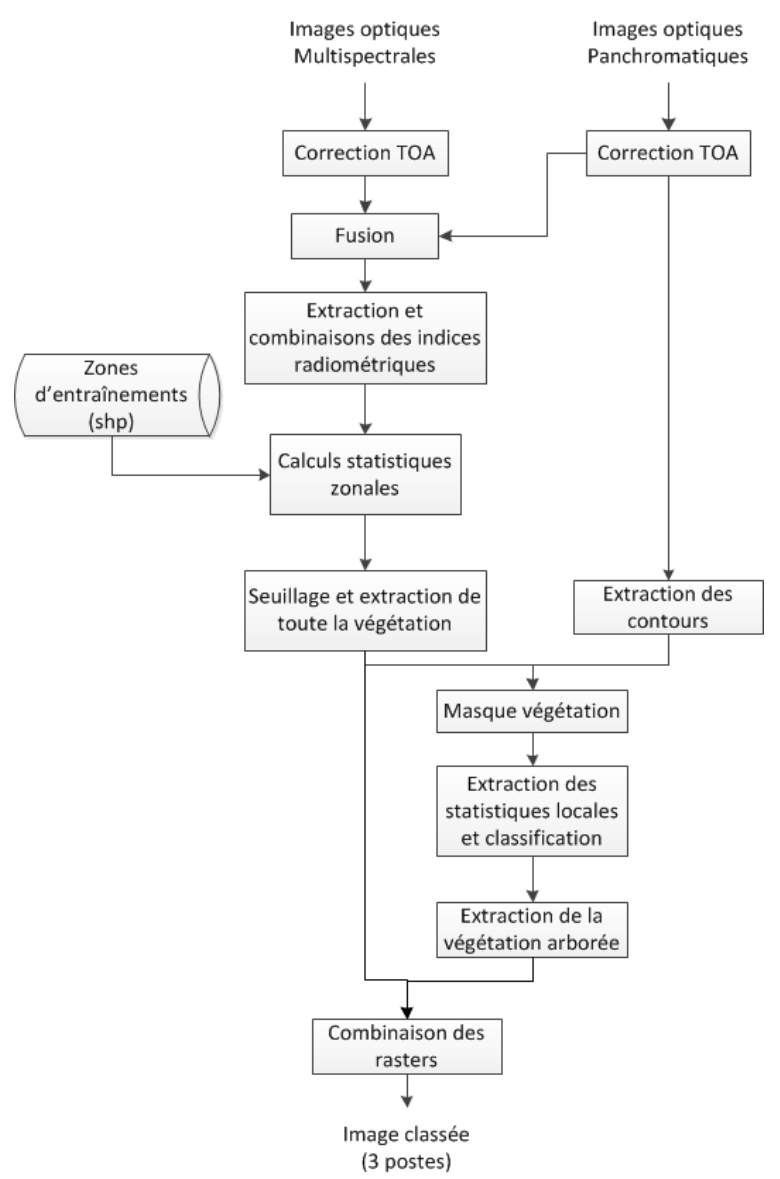

Figure 2 : Chaîne de traitement d'extraction de l'OCS urbaine en 3 postes (Végétation arborée, Végétation herbacée et Autre).

Nous avons réalisé une première correction radiométrique des images en transformant le compte numérique en luminance (correction Top Of Atmosphere (TOA)).

L'approche choisie consiste ensuite à extraire et à combiner deux indices radiométriques. Pour cela, nous calculons pour chaque pixel de l'image l'indice de végétation de la différence normalisée (NDVI) qui permet de cibler la végétation (Rouse et al., 1973). Afin de lever certaines ambiguïtés causées par une réponse spectrale de certains bâtis similaire à celle de la végétation, nous avons calculé un second indice spectral, l'indice de brillance (BI) (Nicoloyanni, 1990) avant de le combiner à l'indice NDVI.

Puis, nous effectuons un seuillage de l'image composite obtenue grâce à une analyse statistique zonale en calculant la valeur moyenne des pixels entièrement inclus dans des zones d'apprentissages. Cette couche de référence a d'abord été basée sur la couche «Végétation » de la BD TOPO® de I'IGN. En analysant les valeurs moyennes obtenues, nous avons confirmé que cette base d'apprentissage ne distinguait que les zones densément végétalisées, à savoir les espaces arborés et les zones plus humides (figure 3-a). Afin d'améliorer l'extraction de la végétation, nous avons donc enrichi cette base d'apprentissage en sélectionnant manuellement de nouvelles zones d'intérêt qui intègrent la végétation basse comme les pelouses. Le résultat de cette première étape de classification est une image binaire (figure 3-b) (végétation/autre) qui servira ensuite de base de connaissance pour le traitement des prochaines images acquises sur la même zone.

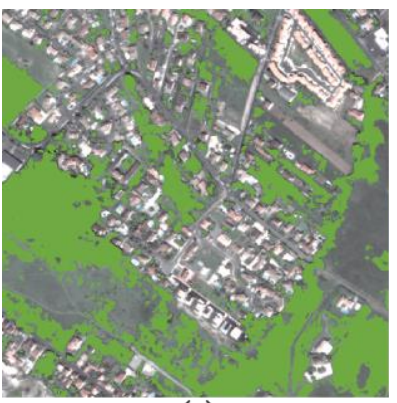

(a)

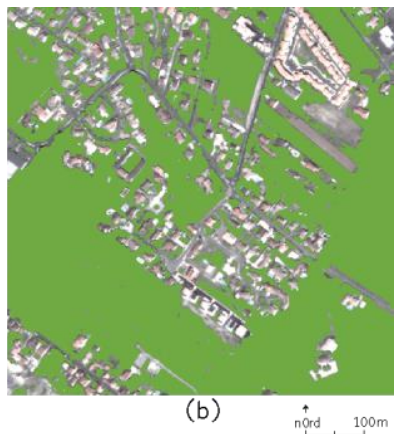

(b)
Végétation (tous types confondus)

Figure 3 : (a) Extraction de la végétation à partir de zones d'entraînements issus de la couche Végétation de la BD TOPO®. (b) Extraction de la végétation à partir de zones d'entraînements sélectionnées manuellement.

Après avoir créé un masque de végétation à partir de la couche de végétation issue de notre classification, les étapes suivantes consistent à discriminer la végétation arborée de la végétation basse. À cet égard, nous avons appliqué un filtre d'extraction des contours sur l'image panchromatique. Cet algorithme associe à chaque pixel de l'image la valeur de la variance locale à l'intérieur d'une fenêtre centrée sur celui-ci. Les valeurs élevées indiquent une texture très prononcée ou correspondent à un contour, elles peuvent être assimilées aux surfaces ligneuses. Après application de ce filtre avec une fenêtre de voisinage d'un rayon de 3 pixels, nous évaluons les valeurs seuils discriminantes entre la végétation basse et arborée par une classification des valeurs obtenues selon la méthode de Jenks.

Enfin, nous avons post-traité ces données dans le but de fournir en complément des données raster une couche vectorielle plus accessible aux utilisateurs finaux. Ces étapes de simplification et de lissage, en lien avec les spécifications et besoins telles que l'Unité Minimale de Collecte (UMC) ou la qualité topologique apportent un gain en termes de maniabilité de la donnée, bien que des limites inhérentes à la vectorisation d'un format raster vont s'observer telles que le crénelage ou la généralisation de la donnée (Bondansky et al., 2002).

Le résultat de cette classification est illustré sur la figure 4 par un extrait cartographique de la végétation urbaine basse et arborée. 


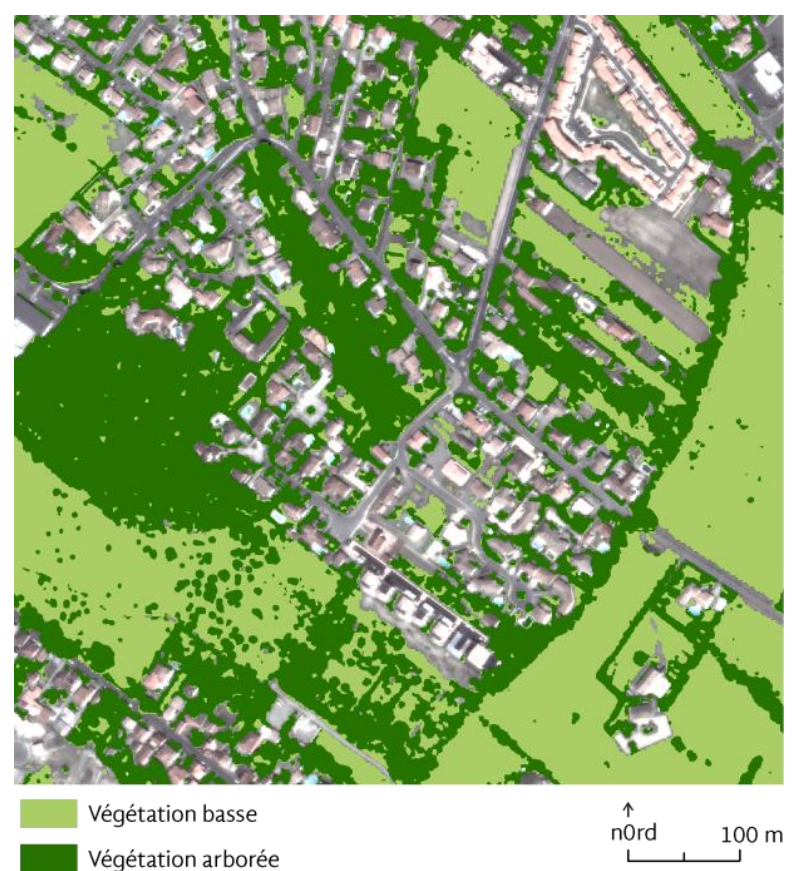

Figure 4 : Extraction de la végétation urbaine arborée et basse.

\section{Comparaisons méthodologique}

Afin d'évaluer nos résultats, nous les avons comparés avec ceux obtenus par (i) une autre méthode de classification d'une image Pléiades (méthode supervisée pixels), (ii) une méthode de classification pixels sur une image SPOT 5 (extraction de la végétation par seuillage d'un indice NDVI) et (iii) la couche vectorielle de végétation de la BD TOPO ${ }^{\circ}$. Pour cela, nous avons sélectionné trois sites d'études dont la densité de population et la surface de bâti varient (centre urbain dense, zone résidentielle dense et zone résidentielle peu dense).

Ces espaces ont été choisis car peu d'évolutions des modes d'occupation des sols sont observées entre 2006 (date de dernière mise à jour de la BD TOPOß) et 2012 (date des images Pléiades et SPOT-5).

La méthode supervisée pixels a été développée par le Centre d'Études et d'Expertise sur les Risques, l'Environnement, la Mobilité et l'Aménagement (CEREMA) pour avant tout répondre à un besoin d'identification fine de la densité urbaine. Elle permet de classer une image THRS Pléiades en quatre postes d'occupation du sol (Bâti, Eau, Route, Végétation) selon une méthode supervisée pixels de type Support Vector Machine (SVM) (Bouffier et Mingam, 2012).

Les résultats quantitatifs sont présentés sur la figure 5 .

Dans l'ensemble, comme l'avait déjà observé Clergeau (2007), nous constatons une évolution croissante de la part de végétation selon un gradient urbain/rural.

Ensuite, ces résultats permettent de confirmer la sousévaluation de la surface végétalisée par la BD TOPO® notamment dans les espaces résidentiels (figure 6). En effet, par définition cette base de données n'identifie que les objets arborés de plus de $500 \mathrm{~m}^{2}$ ou les forêts ouvertes de plus de $5000 \mathrm{~m}^{2}$, omettant donc un grand nombre d'espaces en herbe ou même des jardins publics ou privés qui jouent malgré tout un rôle important dans le maintien de la biodiversité (Marco et al., 2008). Ces insuffisances justifient donc en partie l'usage d'images satellitaires pour cette problématique puisqu'il n'existe pas encore d'autres bases de données vectorielles nationales plus précises que celle de l'IGN.

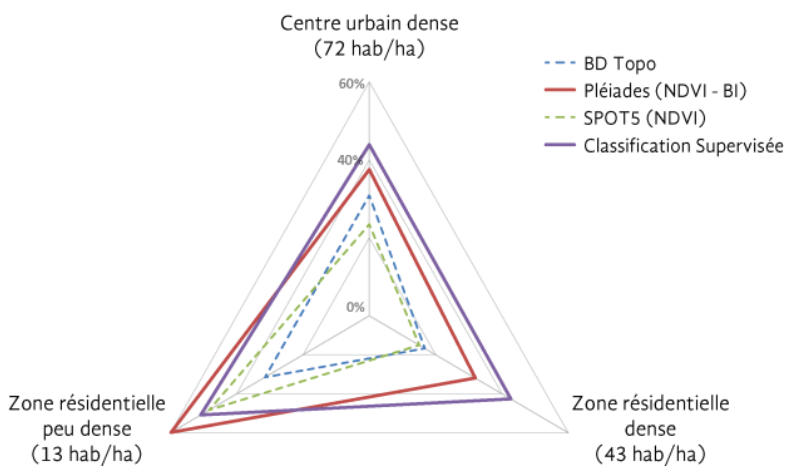

Figure 5: Part de la surface végétalisée par zone d'étude et selon différentes sources de données.

Les résultats obtenus après traitement de l'image SPOT-5 justifient quant à eux l'apport d'une résolution spatiale fine sur ces milieux urbains hétérogènes. En effet, cette hétérogénéité occasionne une réponse spectrale en pixels mixtes (Forster, 1985). Avec une faible résolution spatiale, la création de "mixels ", liée à la végétation qui se confond aux objets urbains, pénalise les traitements et montre tout l'intérêt d'une résolution spatiale fine. Ainsi, la résolution spatiale d'une image SPOT-5 multispectrale étant de $10 \mathrm{~m}$, les petites surfaces de végétation sont moins détectées, d'où la sous-estimation de la part végétale en centre urbain dense. Ce phénomène est moins présent en zone résidentielle peu dense où le bâti devient plus diffus et la végétation plus prégnante.

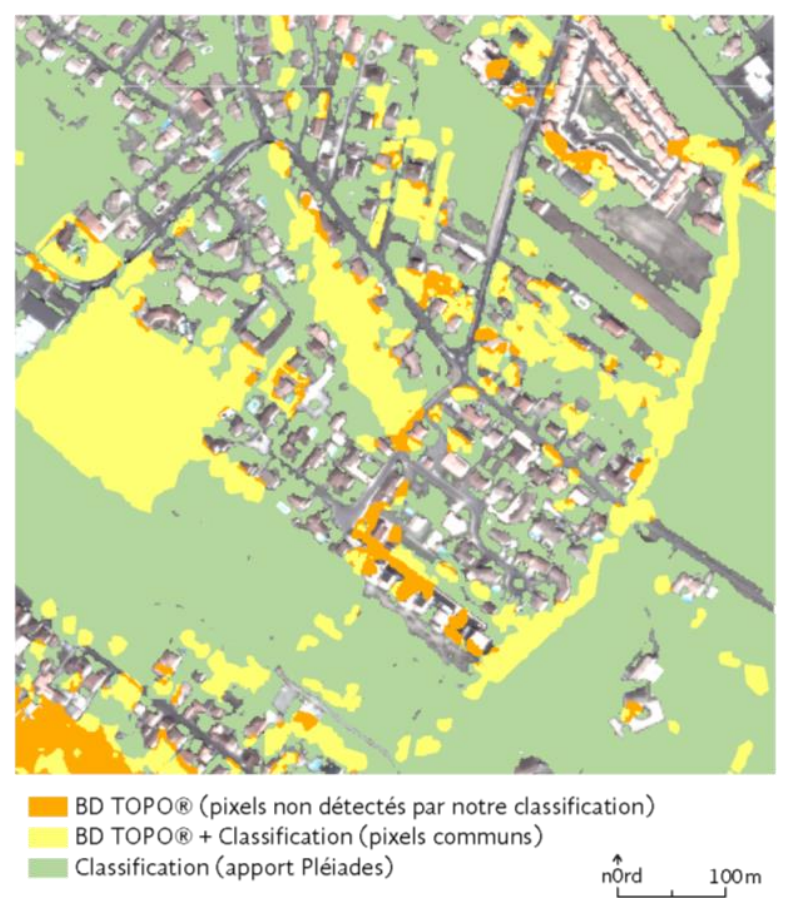

Figure 6: Apport quantitatif de notre méthode de classification comparée à la couche Végétation de la BD TOPO®.

Nos résultats sont proches de ceux obtenus après application de la méthode supervisée pixels du 
CEREMA. Malgré tout, la classe «végétation 》 de leur méthode intègre plusieurs types d'occupation du sol, comme les sols nus que nous ne classons pas en tant que végétation. Cette différence dans la mise en place de la nomenclature peut expliquer la part plus importante de végétation identifiée par la méthode du CEREMA.

Finalement, et pour les besoins identifiés dans cette étude, la méthode que nous avons développée apparait aussi efficace que des méthodes supervisées plus complexes. Par ailleurs, si la méthode orientée objet peut offrir une plus grande diversité thématique dans l'identification de l'occupation du sol, ce gain ne peut être réalisé qu'au prix de nombreux ajustements de paramètres endémiques à la zone étudiée. La méthode proposée ici minimise ces ajustements garantissant une transposabilité aisée tout en satisfaisant aux exigences liées à l'identification d'une trame verte urbaine.

\section{De l'identification à l'évaluation des continuités écologiques}

Les phases évoquées précédemment ont permis d'identifier une couche de végétation qui a ensuite été utilisée pour identifier et évaluer des continuités écologiques urbaines.

Ces dernières se composent de deux éléments au sein d'une matrice paysagère hétérogène (Forman et Godron, 1986): les réservoirs de biodiversité, où les espèces accomplissent la majeure partie de leur cycle de vie, et les corridors écologiques, qui relient les réservoirs de biodiversité et permettent le déplacement des espèces. Ces corridors sont plus difficilement identifiables car encore mal définis scientifiquement, mais ils n'en restent pas moins l'élément majeur d'une connectivité à maintenir. La connectivité des paysages va alors dépendre de deux composantes : (i) un aspect structurel (les taches d'habitats sont-elles géographiquement connectées ou proches les unes des autres ?) et (ii) un aspect fonctionnel (les espèces sont-elles capables d'utiliser un corridor ou même de traverser les mosaïques paysagères moins favorables pour rejoindre d'autres réservoirs de biodiversité ?).

Parmi les avancées récentes en écologie du paysage, nous nous sommes penchés sur l'émergence de travaux de recherche basés sur la théorie des graphes comme ceux de Bunn et al. (2000), Urban et Keitt (2001) ou encore Minor et Urban (2007). Cette approche vise à simplifier et systématiser les territoires complexes en identifiant les connectivités écologiques et en calculant des indices de connectivité pour par exemple évaluer la contribution d'un réservoir de biodiversité à la connectivité totale d'un paysage.

Cette modélisation nécessite deux données d'entrée : (i) une couche identifiant les réservoirs de biodiversité et (ii) un raster de rugosité pour lequel chaque pixel a pour valeur un coefficient de résistance lié à l'occupation du sol. En sortie, nous obtenons une couche de connectivités potentielles, c'est-à-dire des objets linéaires reliant les réservoirs de biodiversité par les chemins plus courts et tenant compte de l'hétérogénéité de la matrice, autrement dit de la difficulté à traverser certains milieux plutôt que d'autres.

Au cours de cette phase, notre première approche a donc consisté à identifier un réseau écologique sur un territoire donné en assimilant les réservoirs de biodiversité aux zones de végétation arborée de plus de deux hectares identifiées lors de la première phase méthodologique. Ce premier paramètre se veut subjectif, non lié à des données écologiques précises mais plutôt à une espèce «fictive ". Là encore cette approche garantit un transfert de la méthode à d'autres espaces. L'idée est bien ici de déterminer des zones à enjeux dont la réalité effective pourra ensuite être affinée par des travaux plus spécifiques mobilisant des données espèces. En l'absence d'observations terrain, nous pouvons utiliser des estimations de capacité de dispersion d'un animal en tant que distance seuil afin de déterminer quels seront les réservoirs connectés. Dans notre exemple, aucune valeur seuil n'est utilisée. Ainsi, nous cherchons à modéliser l'ensemble des chemins de moindres coûts reliant tous les réservoirs de biodiversité entre eux.

Nous élaborons ensuite le second paramètre d'entrée, une carte de rugosité obtenue en tenant compte de l'occupation du sol (figure 7). Moins le milieu est végétalisé, plus il est difficilement franchissable. Puis nous affectons un coefficient croissant en fonction de la largeur des routes et de la hauteur des bâtis. Ces données attributaires sont obtenues à partir de la BD TOPO® de I'IGN, seule base de données disposant facilement et rapidement de cette information, et sont ensuite rééchantillonnées sur une échelle de 0 à 100.

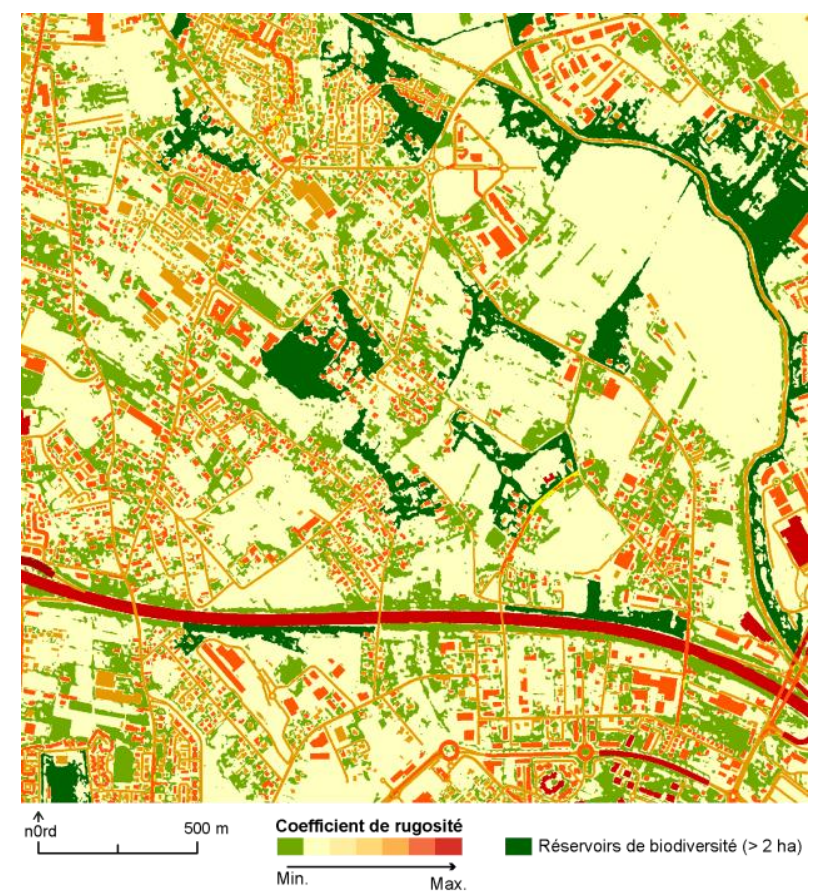

Figure 7 : Carte des coefficients de rugosité sur la zone test de $9 \mathrm{~km}^{2}$.

En superposant par exemple ce réseau aux zones classées «à urbaniser » du PLU, nous identifions alors, au centre de notre territoire d'étude, des zones conflictuelles où des espaces urbanisables recouvrent des réservoirs de biodiversité ainsi que des corridors écologiques potentiels (figure 8). Ce sont plus particulièrement ces zones-là qui nécessiteront une attention particulière dans les projets d'aménagements urbains. 


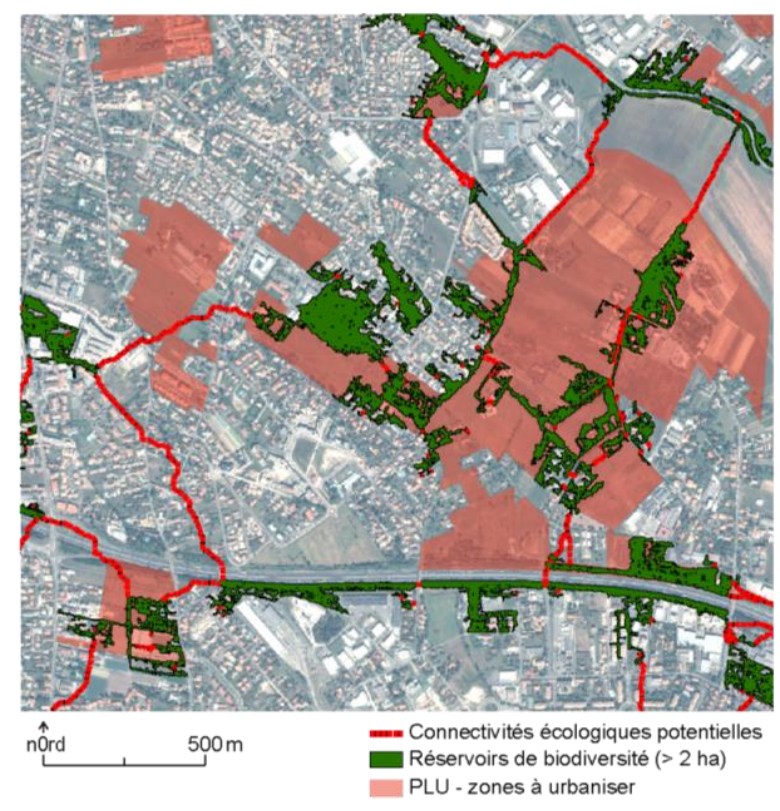

Figure 8 : Mise en évidence de zones à enjeux par superposition du réseau écologique et des zones à urbaniser du PLU.

\section{Perspectives}

L'évaluation d'une autre méthode supervisée faisant appel à plus d'étapes de classification est actuellement en cours. L'objectif affiché ici est de mesurer l'efficacité des méthodes selon des critères de qualité des résultats, de temps de traitement et de coût financier engagé (matériel informatique, logiciels ou encore formation du personnel).

\section{Conclusion}

Sur les territoires à forte urbanisation, la compétition entre l'urbanisation et la conservation de la biodiversité est bien présente. II est donc nécessaire de mettre en place des outils d'évaluation des connectivités écologiques reflétant les besoins de cohésion des systèmes urbains et la diminution des effets négatifs de la fragmentation des territoires sur la biodiversité.

Ce papier a détaillé dans un premier temps une chaîne de traitement utilisant une image satellite optique Pléiades à très haute résolution spatiale pour l'identification de la végétation urbaine.

II a ainsi été montré que ces images satellitaires constituent une source de données principale pour l'extraction de la végétation urbaine et apportent un avantage certain en termes de résolution spatiale, un critère exigé lors d'une analyse détaillée des villes, notamment dans les zones périurbaines dynamiques. Cette méthodologie a été développée à l'aide d'outils libres comme l'OTB pour les traitements d'images raster, le logiciel QIS pour l'analyse spatiale des données géographiques et le logiciel Graphab pour la mise en application de la théorie des graphes.

Les résultats correspondent à l'application visée et pourront être affinés par l'ajout d'autres données comme celles issues d'inventaires faunistiques ou floristiques. Les lecteurs doivent garder à l'esprit que les indices de rugosité utilisés pour définir la donnée d'entrée du modèle peuvent être modifiés et adaptés à la situation ou à des espèces précises. De plus, modifier la qualité et la résolution spatiale des données d'entrée peuvent également engendrer des différences significatives entre les cartes de connectivités potentielles. Les limites restreintes de cette zone d'étude ont été sélectionnées pour faciliter la mise en application de cette méthode. Cependant, la sélection pourra également s'appuyer sur des priorités politiques d'aménagement ou sur des aspects socio-économiques.

\section{Remerciements}

Ce travail de recherche a été réalisé avec le soutien financier du CNES et de la Région Midi-Pyrénées. Les auteurs remercient le CNES pour la mise à disposition des images satellites SPOT5 et Pléiades (C) CNES (2012), distribution Airbus DS / Spot Image)) via la RTU et la base de données Kalideos "OSR MiPy » ainsi que le CEREMA pour sa contribution dans le cadre de la comparaison des méthodes de classification d'images.

\section{Références}

Baudry J., Merriam G., 1988. Connectivity and connectedness: functional vs structural patterns in landscapes. In Proceedings of the 2nd Seminar of the International Association for Landscape Ecology, Munster, 29, 23-28.

Bondansky E., Gribov A., Pilouk M., 2002. Smoothing and Compression of Lines Obtained by Raster-toVector Conversion. In Blostein D. et Kwon Y.-B. (dir.), Graphics Recognition Algorithms and Applications, Springer Berlin Heidelberg, 256-265.

Bouffier J., Mingam B., 2012. Apport potentiel de l'imagerie satellitaire pour la caractérisation fine de la densité urbaine CETE SO/DALETT/SCGSI, 10p.

Bunn A. G., Urban D.L., Keitt T.H., 2000. Landscape connectivity: A conservation application of graph theory. Journal of Environmental Management, 59(4), 265-278.

Clergeau P., 2007. Une écologie du paysage urbain. Editions Apogée, Paris. 137p.

Clergeau P., Hubert-Moy L., 2011. L'émergence d'une problématique scientifique en relation avec une demande sociétale. In Clergeau P. (dir.), Ville et biodiversité; Les enseignements d'une recherche pluridisciplinaire. Presses Universitaires de Rennes. 235p.

Forman R. T. T., Godron M., 1986. Landscape ecology. John Wiley and Sons, New-York. 640p.

IGN, 2011. BD TOPO® Version 2.1 Descriptif de contenu. 172p.

Forster B. C., 1985. An examination of some problems and solutions in monitoring urban areas from satellite platforms. International Journal of Remote Sensing, 6(1), 139-151. 
Marco A., Dutoit T., Deschamps-Cottin M., Mauffrey J. F., Vennetier M., Bertaudière-Montes V., 2008. Gardens in urbanizing rural areas reveal an unexpected floral diversity related to housing density. Comptes Rendus Biologies, 331(6), 452-465.

Minor E. S., Urban D., 2007. A graph-theory framework for evaluating landscape connectivity and conservation planning. Conservation Biology, 22(2), 297-307.

Nicoloyanni E., 1990. Un indice de changement diachronique appliqué à deux scènes Landsat MSS sur Athènes (Grèce). International Journal of Remote Sensing, 11(9), 1617-1623.

Rouse J.W., Haas R.H., Schell J.A., Deering D.W., 1973. Monitoring vegetation systems in the great plains with ERTS, Third ERTS Symposium, NASA SP-351 I: 309-317.

Uezu A., Metzger J. P., Vielliard J. M. E., 2005. Effects of structural and functional connectivity and patch size on the abundance of seven Atlantic Forest bird species. Biological Conservation, 123(4), 507-519.

Urban D., Keitt T., 2001. Landscape connectivity: A graph-theoretic perspective. Ecology, 82(5), 1205-1218. 